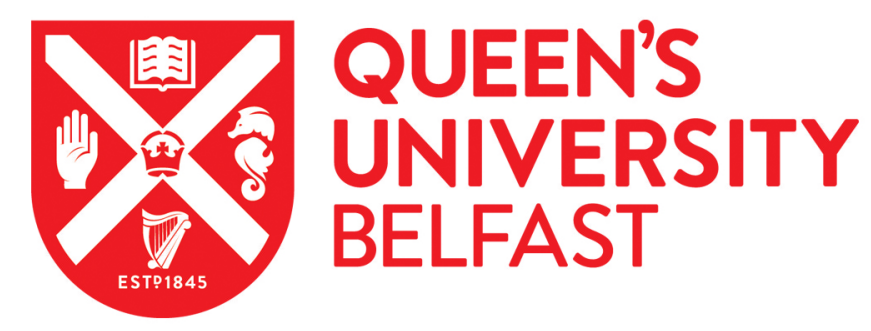

\title{
Recent developments in the Thomson Parabola Spectrometer diagnostic for laser-driven multi-species ion sources
}

Alejo, A., Gwynne, D., Doria, D., Ahmed, H., Carroll, D. C., Clarke, R. J., Neely, D., Scott, G. G., Borghesi, M., \& Kar, S. (2016). Recent developments in the Thomson Parabola Spectrometer diagnostic for laser-driven multispecies ion sources. Journal of Instrumentation, 11, [C10005]. https://doi.org/10.1088/1748-0221/11/10/C10005

Published in:

Journal of Instrumentation

Document Version:

Peer reviewed version

Queen's University Belfast - Research Portal:

Link to publication record in Queen's University Belfast Research Portal

Publisher rights

(c) 2016 IOP Publishing.

This work is made available online in accordance with the publisher's policies. Please refer to any applicable terms of use of the publisher.

\section{General rights}

Copyright for the publications made accessible via the Queen's University Belfast Research Portal is retained by the author(s) and / or other copyright owners and it is a condition of accessing these publications that users recognise and abide by the legal requirements associated with these rights.

Take down policy

The Research Portal is Queen's institutional repository that provides access to Queen's research output. Every effort has been made to ensure that content in the Research Portal does not infringe any person's rights, or applicable UK laws. If you discover content in the Research Portal that you believe breaches copyright or violates any law, please contact openaccess@qub.ac.uk. 


\title{
Recent developments in the Thomson Parabola Spectrometer diagnostic for laser-driven multi-species ion sources
}

\author{
A. Alejo, ${ }^{a}$ D. Gwynne, ${ }^{a}$ D. Doria,${ }^{a}$ H. Ahmed, ${ }^{a}$ D.C. Carroll, ${ }^{b}$ R.J. Clarke, ${ }^{b}$ D. Neely, ${ }^{b}$ G.G. \\ Scott, ${ }^{b}$ M. Borghesi ${ }^{a}$ and S. Kar ${ }^{a, 1}$ \\ ${ }^{a}$ Centre for Plasma Physics, School of Mathematics and Physics, Queen's University Belfast, Belfast BT7 \\ INN, United Kingdom \\ ${ }^{b}$ Central Laser Facility, Rutherford Appleton Laboratory, Didcot OX11 OQX, United Kingdom \\ E-mail: s.kar@qub.ac.uk
}

ABSTRACT: Ongoing developments in laser-driven ion acceleration warrant appropriate modifications to the standard Thomson Parabola Spectrometer (TPS) arrangement in order to match the diagnostic requirements associated to the particular and distinctive properties of laser-accelerated beams. Here we present an overview of recent developments by our group of the TPS diagnostic aimed to enhance the capability of diagnosing multi-species high-energy ion beams. In order to facilitate discrimination between ions with same $\mathrm{Z} / \mathrm{A}$, a recursive differential filtering technique was implemented at the TPS detector in order to allow only one of the overlapping ion species reaching the detector, across the entire energy range detectable by the TPS. In order to mitigate the issue of overlapping ion traces towards the higher energy part of the spectrum, an extended, trapezoidal electric plates design was envisaged, followed by its experimental demonstration. The design allows achieving high energy-resolution at high energies without sacrificing the lower energy part of the spectrum. Finally, a novel multi-pinhole TPS design is discussed, that would allow angularly resolved, complete spectral characterization of the high-energy, multi-species ion beams.

Keywords: Spectrometers, Plasma diagnostics - charged-particle spectroscopy, Plasma generation (laser-produced, RF, x ray-produced), Ion identification systems

\footnotetext{
${ }^{1}$ Corresponding author.
} 


\section{Contents}

1 Introduction 1

2 Basics of the Thomson Parabola Spectrometer 2

3 Differential Filtering 3

4 Trapezoidal Plates $\quad 5$

5 Multi-pinhole TPS $\quad 6$

6 Conclusions $\quad 8$

\section{Introduction}

For over a decade, the field of laser-driven ion acceleration has attracted significant attention due to the potential compactness and cost-effectiveness associated with a laser-based approach, which can facilitate a range of applications [1]. The most studied ion acceleration mechanism so far has been the Target Normal Sheath Acceleration (TNSA)[2-4], capable of providing energetic, multispecies ion beams, mainly accelerating light ions $\left(\mathrm{H}^{+}, \mathrm{C}^{n+}, \mathrm{O}^{n+}\right)$ from the contamination layer covering the target $[5,6]$. Commonly used diagnostics for characterising such ion beams include radiochromic film stacks [7], nuclear activation [8], nuclear track detection [9, 10], which are in general capable of providing spatial and spectral information about an ion beam (See e.g. Ref. [11] for a comprehensive overview of detection methods). However, in presence of a multi-species ion beam dominated by protons, as typically obtained from laser driven sources, these diagnostics mainly provide information about the protons due to their lowest stopping power amongst all ion species. The Thomson Parabola Spectrometer (TPS)[12] is therefore used in most laser-plasma experiments due to its unique capability of simultaneously characterising the energy spectrum while separating ion species with different charge (Z) - to - mass (A) ratios. Recent evolutions in laser technologies leading to currently available laser intensities above $10^{20} \mathrm{~W} \mathrm{~cm}^{-2}$, has also opened up the possibility of accessing new acceleration mechanisms, such as Radiation Pressure Acceleration (RPA)[13-16] or Break-Out Afterburner (BOA)[17-19], which are, in principle, capable of accelerating bulk target ions to significant energies (towards $100 \mathrm{MeV} /$ nucleon range). Such enhanced beam characteristics pose a challenge for the TPS as originally designed. In this paper we present a review of the recent improvements brought to the design of Thomson Parabola Spectrometers, trying to overcome their limitations in terms of characterising the high-energy, multi-species ion beams produced at high power laser facilities. 


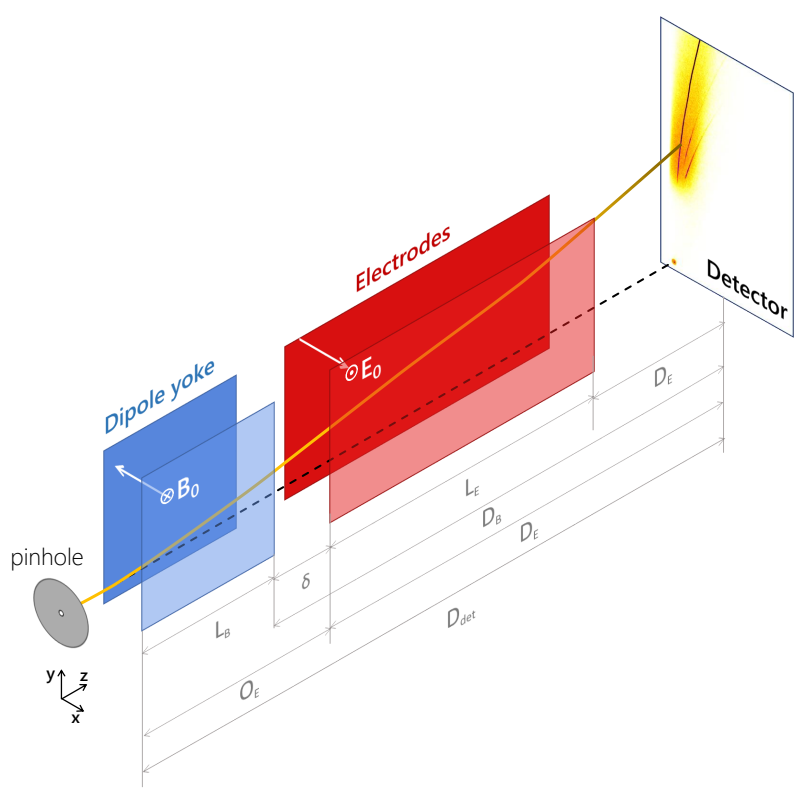

Figure 1. Schematic of a typical TPS with regions of static magnetic and electric fields. The trajectories of a positively-charged ion and a neutral particle are depicted by a yellow line and a dashed black line, respectively.

\section{Basics of the Thomson Parabola Spectrometer}

Thomson Parabola Spectrometers [12] have the unique ability to energy-resolve ion spectra while discriminating ions with different charge-to-mass ratios, thanks to the combined use of electric and magnetic fields. In a typical TPS (see Fig. 1), a pencil beam of ions, selected by the pinhole located at its entrance, travels through regions of parallel magnetic and electric fields applied transversely to the beam axis. In reference to Fig. 1, the magnetic field determines the $y$-coordinate of the ions on the detector, depending on their energy, while the electric field deflects the ions along the $x$-axis according to their Z/A ratio. Assuming that the electric and magnetic fields are uniform (with inhomogeneities such as fringe effects being negligible), the coordinates of the ions on at the detector plane along the $y$ (owing to the magnetic field) and $x$ (owing to the electric field) axes can be derived analytically [20] as stated below,

$$
\begin{aligned}
& x=\frac{\gamma}{\gamma^{2}-1} \frac{q E_{0} L_{E}}{m_{0} c^{2}}\left(D_{E}+L_{E} / 2\right), \\
& y=\frac{1}{\sqrt{\gamma^{2}-1}} \frac{q B_{0} L_{B}}{m_{0} c}\left(D_{B}+L_{B} / 2\right),
\end{aligned}
$$

where $c$ is the speed of light, $m_{0}$ and $q$ are the rest mass and charge of the ions, $E_{0}$ and $B_{0}$ are the electric and magnetic fields, $L_{B}, D_{B}, L_{E}$ and $D_{E}$ are the dimensions of relevant sections of the TPS as labelled in Fig. 1a, and $\gamma$ is the relativistic Lorentz factor.

For non-relativistic speeds $\left(v_{z} \ll c\right)$, the general expression can be approximated by performing a first-order Taylor expansion around $\gamma=1$. Introducing the kinetic energy $\left(E=[\gamma-1] m_{0} c^{2}\right)$, one can obtain the formulae matching with the non-relativistic equations commonly used for this 
diagnostic $[21,22]$.

$$
\begin{gathered}
x=\frac{q E_{0}}{2 E} L_{E}\left(\frac{1}{2} L_{E}+D_{E}\right), \\
y=\frac{q B_{0}}{\sqrt{2 m_{0} E}} L_{B}\left(\frac{1}{2} L_{B}+D_{B}\right),
\end{gathered}
$$

where particles of a given $q / m$ lay on a parabolic trace described by the expression

$$
y^{2}=\frac{q}{m_{0}} \frac{B_{0}^{2}}{E_{0}} \frac{L_{B}^{2}\left(L_{B} / 2+D_{B}\right)^{2}}{L_{E}\left(L_{E} / 2+D_{E}\right)} \cdot x
$$

Where most of the physical parameters of the TPS can be easily measured, it is paramount to map the magnetic field accurately, whether using permanent magnets or electromagnets [23-25], accounting in particular for non-uniformities in the field along the beam axis. The magnetic field inside a yoke can either be mapped empirically using a Hall probe [25, 26], or modelled numerically as described in Refs. [27-29]. The signal obtained on the detector along a given parabolic ion trace can be converted to a raw ion spectrum using the Eqs. 2.1, 2.2. However, to obtain an absolute ion spectrum, a calibration from the signal to ion numbers would be required, which depends on the type of the detector and the ion species of concern. Since the response of the detectors for different charged particles depends heavily on the ion species and energy, calibration functions for the most common types of TPS detectors and particles have been obtained experimentally by different groups. Some of the relevant works include calibration of Image Plates [30] for protons [31-33], deuterons [34, 35], alpha particles [35, 36] and carbon ions [37], and the calibration of MultiChannel Plates for protons [23, 38] and carbon ions [39].

Although TPSs are commonly used and are clearly suitable for the characterisation of multispecies laser-driven ion sources, there are three main limitations compromising their use in experiments. As it can be realised from Eq. 2.5, (1) The locus of the parabolic ion traces produced by the TPS is a function of Z/A. Therefore, the traces of species with the same $Z / A$ will be overlapped at the detector plane, preventing their spectra to be analysed. (2) The separation between the traces not only depends on Z/A but also on the ion energy. For high energies, the small deflection of the ions by the fields leads to merging of the traces even for ions with different $Z / A$. (3) Finally, in order to have a pencil beam of ions, a pinhole has to be placed at the front of the TPS, which forbids obtaining any spatial information about the ion beam. This is particularly a concern where the ion beams do not exhibit a uniform spatial distribution, which is a characteristic of some laserdriven acceleration mechanisms. The modifications to the original TPS design to overcome the aforementioned limitations are discussed in the following sections.

\section{Differential Filtering}

As mentioned above, the parabolic traces of ion species with the same $Z / A$ will overlap at the detector plane, preventing their spectra to be characterised. This particularly concerns targets containing deuterium, which are used for studies related to the development of compact laserdriven neutron sources [40]. In this case, the trace for $\mathrm{D}^{+}$ions overlaps with the traces of ions produced by fully ionising the other atoms, such as carbon and oxygen, present in the hydrocarbon 
and water vapour contaminant layers. Therefore, the deuteron spectra cannot be analysed unless one blocks the overlapping $\mathrm{C}^{6+}$ and $\mathrm{O}^{8+}$ species reaching the detector.

In order to detect the lightest of the overlapping ion species, one can consider placing a filter in front of the detector, which would preferentially stop heavier ions and allow only lighter species to be detected (due to the differences in the stopping powers of different ion species in a given material [41]). However, due to the broad energy spectrum of the laser-driven ions (ranging from sub-MeV up to tens of $\mathrm{MeV}$ ), a single filter would either be too thin to stop the heavy ions of high energies, or too thick to let the light ions of low energy pass through. In order to overcome this limitation, one can use a stepped Differential Filtering (DF) technique [34], which consists of an array of foils of different materials and thickness along the energy dispersion $(y)$ axis, ensuring the discrimination of the lightest ions from the rest of the overlapping species is achieved in the energy range covered by each filter.
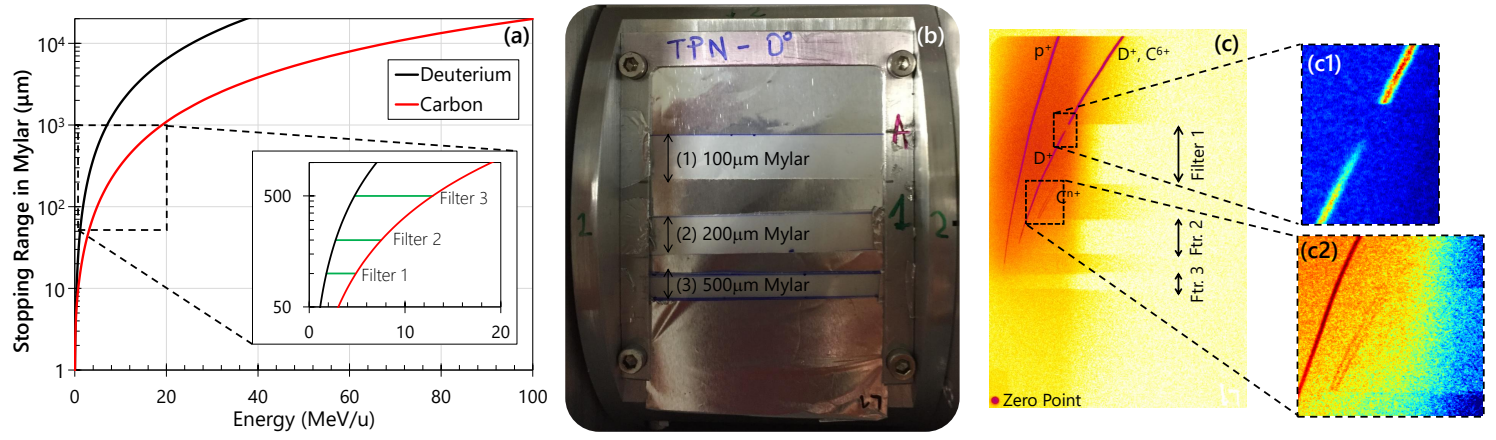

Figure 2. (a). Stopping range of deuterium (black) and carbon (red) ions in mylar, as obtained using SRIM[41]. The dashed rectangle corresponds to the area plotted in the inset, where the energy ranges in which the green lines depict the energy range in which a $100 \mu \mathrm{m}, 200 \mu \mathrm{m}$ and $500 \mu \mathrm{m}$ mylar foil can be used. (b) Picture of the experimental configuration, with a Differential Filter mounted on an aluminium frame and placed over a detector. (c) Measured signal for the detector shown in (b), with shadows appearing in the areas were the filter was present, and heavier species appearing in the non-filtered regions. (c-1), (c-2) Zoomed-in views of the detector showing the gap on the ion trace and the appearance of heavier species between the filtered regions.

The choice for the thickness and material for each filter can vary depending on the materials available and the species to be discriminated. As an example, the discrimination of $\mathrm{D}^{+}$from $\mathrm{C}^{6+}$ ions is discussed here, which was tested in an experiment. At a given point on the parabolic trace, the overlapping ion species possess the same energy per nucleon. This is a favourable condition for differential filtering as the stopping range of ions of different masses can vary significantly at a given energy per nucleon. Therefore a filter of thickness greater than the stopping range of the heavier ions, and smaller than the stopping range of the lighter species can be used as a differential filter at the given location on the trace. The stopping ranges of $\mathrm{p}^{+}$and $\mathrm{C}^{6+}$ ions in mylar are shown in Fig. 2(a), where a mylar foil of a given thickness can filter the energies between the two curves.

In our case, deuterium ions with energies from 2 to $15 \mathrm{MeV} / \mathrm{n}$ were diagnosed by the TPS. The ion beam was produced by irradiating the petawatt arm of the Vulcan laser [42] to $25 \mu \mathrm{m}$ thick foils made of deuterated plastic (CD). The TPS was placed along the target normal axis, at a distance 
$\sim 1 \mathrm{~m}$ from the target. Mylar foils of thickness $100 \mu \mathrm{m}, 200 \mu \mathrm{m}$ and $500 \mu \mathrm{m}$ (refereed here as filter 1 , filter 2 and filter 3 in this paper) were chosen for the different sections of the spectrum, covering an energy range shown by the green lines in Fig. 2(a). A DF was designed using these foils while taking into account the specific parameters of our TPS. Fig. 2(b) shows the picture of the DF used in the experiment, where the filters were mounted on an aluminium frame that holds the detectors, thereby avoiding any vertical displacement of the filters with respect to the detector.

Fig. 2(c) shows the data obtained on the image plate detector placed behind the DF. As one can see, clear shadows appeared in those regions where the filters were present. The large gaps between the filters were intentionally introduced, so that the filtered regions will allow the deuterium spectrum to be obtained, while the $\mathrm{C}^{6+}$ spectrum can be calculated by subtracting the interpolated deuteron-only signal from the total $\left(\mathrm{C}^{6+}\right.$ and $\left.\mathrm{D}^{+}\right)$signal. The Filter-1 in this case was placed a few $\mathrm{mm}$ above its calculated height. Therefore, as can be seen in the Fig. 2(c) and inset (c-1), the ion signal abruptly disappeared as the Filter-1 clips the ion spectrum. The signal started to reappear after a few $\mathrm{mm}$ down in the filter, as the higher energy deuterons start to penetrate the filter. One can also see a small drop of signal in the ion trace within the Filter-1 region compared to the ion trace above the Filter-1, which is most likely due to filtering of the carbon ions by the Filter-1. The effectiveness of differential filtering can also be seen from the disappearance of $\mathrm{C}^{4+}$ and $\mathrm{C}^{5+}$ traces in the regions covered by the filters (Fig. 2(c-2)). This technique has been successfully implemented in a number of experiments studying laser driven deuterons produced under different conditions $[40,43]$.

\section{Trapezoidal Plates}

As mentioned before, a DF can be used to discriminate between species with the same $Z / A$ ratio overlapping on the detector. However, for the case of high-energy ion sources, the different species can also overlap at high ion energies, even when they possess different $Z / A$, due to the small deflection of the energetic particles by the fields. The traces for two given species will merge together when the width of the traces, defined by the size of the pinhole, is larger than the separation between them [21,44]. Analytically, this separation can be expressed as

$$
\begin{aligned}
\delta=\left|x_{2}-x_{1}\right|_{y_{2}=y_{1}} & =\frac{Z_{2} e E_{0} L_{E}\left(D_{E}+L_{E} / 2\right)}{A_{2} m_{p} c^{2}} \frac{\gamma_{2}}{\gamma_{2}^{2}-1}\left|1-\frac{1}{\gamma_{2}} \sqrt{\gamma_{2}^{2}-1+\left(\frac{A_{1}}{A_{2}} \frac{Z_{2}}{Z_{1}}\right)^{2}}\right| \\
& \longrightarrow \frac{Z_{2} e E_{0} L_{E}\left(D_{E}+L_{E} / 2\right)}{2 E_{2}}\left|\frac{A_{1}}{A_{2}} \frac{Z_{2}}{Z_{1}}-1\right|
\end{aligned}
$$

where $\left\{Z_{1}, A_{1}\right\}$ and $\left\{Z_{2}, A_{2}\right\}$ are the atomic and mass numbers of each ion species, and $E_{2}, \gamma_{2}$ are the kinetic energy and Lorenz factor of one of them.

In light of Eq. 4.1, there are three ways to increase the separation between the two traces: (1) Increasing the electric field, (2) enlarging the electrode length $\left(L_{E}\right)$ [21, 22], or (3) placing the detector farther away $\left(D_{E}\right)$ [22]. However, the increase in $D_{E}$ in most cases is limited by the size of the vacuum chambers, where the diagnostic has to be placed, whereas the maximum electric field attainable is limited by the vacuum conditions inside the chamber $(\sim 20 \mathrm{kV} / \mathrm{cm}$ is typically attainable in a laser-plasma interaction chamber at $\sim 10^{-4} \mathrm{mbar}$ ). Therefore, the most pragmatic 
route for improving the separation between the ion traces is increasing the length of the electrodes, which will lead to a quadratic improvement in the trace separation with the increase in the length of the electrode.

Unfortunately, the increase in the electrode length also causes the lower energy part of the ion spectrum to intercept the electric plates due to their large dispersion, which prevents their detection. In order to allow transmitting low energy particles to the detector in an extended electrode case, one may use a wedged configuration as studied in Refs. [26, 28]. However, an increase in separation between the plates in this configuration reduces the field strength commensurately, compromising the net separation between neighbouring species at high ion energies. An alternative method is using trapezoidally shaped electric plates, as suggested by Gwynne et al. [21]. With such electrodes, the high-energy ions will effectively experience a longer electric field region, while the slower ions will be able to reach the detector by passing over the wedged side of the electrode. The effectiveness of the trapezoidally shaped electrodes was thoroughly investigated by Gwynne et $a l$. by varying the electrode dimension and shape in fully relativistic particle tracing simulations. The design was recently tested experimentally [20]. In order to increase the maximum resolvable energies for proton and carbon ions, $30 \mathrm{~cm}$-long trapezoidal electrodes were used in the experiment, which is double the length of the electrodes simulated in [21]. As a result, the separation between neighbouring traces was increased significantly for the high-energy ions (extending the maximum resolvable carbon energy up to $350 \mathrm{MeV}$ ), without clipping the low energy part of the spectrum. The experimental data was found to be in an excellent agreement with an analytical model, which does not take into account the electrostatic fringe fields above the wedged side of the electrodes. As suggested by simulations in Ref. [21], the experimental data shown by Alejo et al. in Ref. [20] indicates negligible fringe field effects on the low energy ions escaping over the wedged side of the electrode, which simplifies significantly the data analysis.

\section{Multi-pinhole TPS}

The third limitation of a TPS discussed in this paper is its inability to resolve the ion spectrum angularly, since it requires a pencil beam of ions at its entrance. Stacks of Radiochromic film (RCF) detectors can be used to obtain an angularly-resolved, coarse spectra for protons, although spectral analysis using RCF data has limitations due to the discrete energy sampling allowed by consecutive RCF layers in a stack $[7,45]$. However, a comprehensive characterisation of multi-species ion beams is not possible by using such approach. The minimum achievable angular resolution by deploying two TPSs close to each other can be 5 - 10 degrees, depending on how far from the source one can place the TPS with sufficient ion flux for detection. The lack of information about the angular dependence of the ion spectra is of particular concern in cases of highly structured beam, as often produced, for example, from the interactions of intense lasers with ultrathin foils [15, 46].

Some efforts have been made in order to increase the solid angle of detection by using magnetic spectrometers with large acceptance angles, such as the Imaging Proton Spectrometer [47] and the iWASP [48], in which the pinhole is substituted by a horizontal slit. By placing a filter in front of the detector, an angularly-resolved proton spectrum can be obtained with significantly higher energy resolution than RCF stacks. However, the absence of electric field deflection forbids a full characterisation of multi-species ion beams. In an attempt to improve this diagnostic, Senje et al. 
[49] developed a modified iWASP, in which an ion beamlet was selected by using an vertical slit after the magnetic field and applying an electric field to the beamlet for detailed spectral characterisation. This design allows for simultaneous characterisation of the angularly-resolved proton spectrum and full spectral characterisation of different species along one specific direction. A multipinhole approach applied to a Thomson parabola was developed in [50, 51], where several adjacent pinhole (obtained in the same substrate) were located at the entrance of a standard TPS. This set-up allowed discrete angular sampling of the beam, although only within a restricted angular range ( $\sim 2$ degrees).

Here we present a novel design for a compact, multi-pinhole (MP) TPS (Fig. 3) which, combining some of the elements described above, would be capable of capturing fully resolved ion spectra along multiple directions, and over an extended angular range of 30 degrees. In this design, the entrance pinhole is substituted by a horizontal slit that selects ions emitted within a broad angle, with an angled dipole yoke spreading the particles along the $y$ direction depending on their energy, similar to the first stage of an iWASP. A set of vertical slits is placed after the magnet, where each of the vertical slits is coupled with the input horizontal slit to perform angular selection similar to the input pinhole in a standard TPS design. The ions passing through each vertical slit will be able to travel through an electric field region created by a pair of electrodes. In order to have a strong field perpendicular to the ion axis, each slit would require its own different set of electrodes. Since the sets of electrodes will have to be placed close to each other, in order to maximise the angular resolution, one can alternate the electric field direction in the consecutive sets of electrodes. With the same voltage being applied to adjacent electrodes between two sets of plates, this design will allow deploying high electric fields across the ion beams.

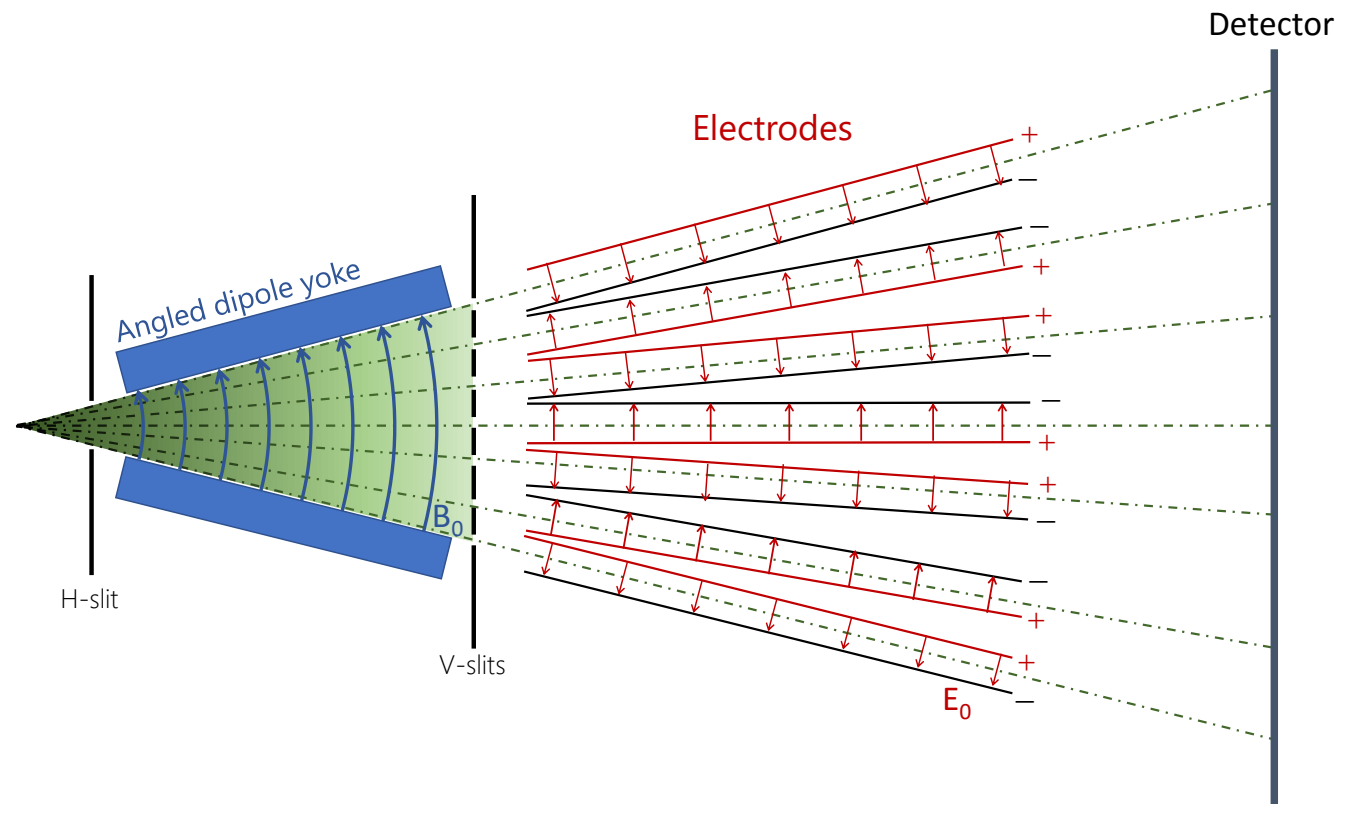

Figure 3. Schematic top view of a large collection angle Multi-Pinhole TPS. The characteristics of the dipole yoke can be found in [48], with a length of $100 \mathrm{~mm}$. Each set of electrodes consists of two metal plates with length of $150 \mathrm{~mm}$ and separated by $10 \mathrm{~mm}$, where the electric field applied is $16 \mathrm{kV} / \mathrm{cm}$. 
Although a more complex design can allow sampling at smaller angular intervals, a test design consisting of 14 electric plates, allowing for the simultaneous measurement along 7 angles (Fig. 3), was simulated using the SIMION particle tracing code[52]. Similar parameters as those commonly employed in experiments were used in the simulations. The characteristics for the dipole yoke were taken from Ref. [48] and $150 \mathrm{~mm}$-long electric plates were used with $E_{0}=16 \mathrm{kV} / \mathrm{cm}$. The simulated ion traces on the detector are shown in Fig. 4, where the $\mathrm{p}^{+}$and $\mathrm{C}^{6+}$ ions are shown in blue and red, respectively. One can see the ion parabolas in neighboring angular sets are reversing their orientation, as a consequence of the alternating field polarity.

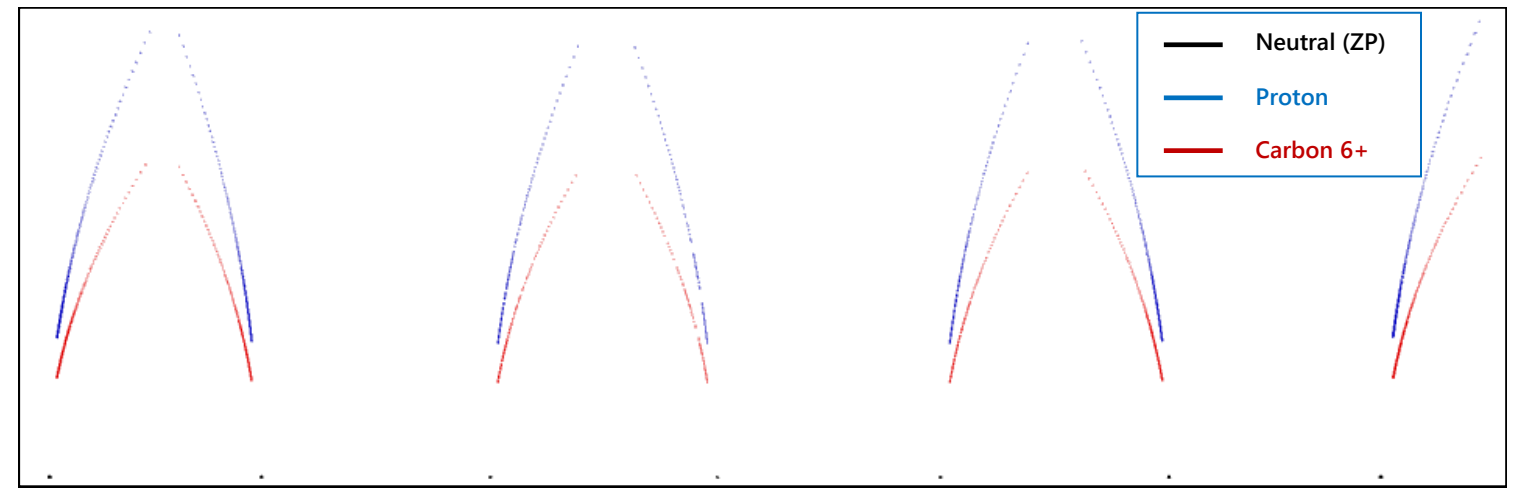

Figure 4. Results for a particle tracing simulation showing the position on the detector of $\mathrm{p}^{+}$(blue), $\mathrm{C}^{6+}$ (red) and neutral (black) particles.

\section{Conclusions}

The interest in characterising different species makes the Thomson Parabola Spectrometers a natural choice, although improvements on the traditional design are needed in order to diagnose the beam expected at the current/upcoming laser facilities. Possible solutions to overcome three main issues with these diagnostics are discussed. The use of differential filters enables the characterisation of the lightest ions from a set of ion species with same charge-to-mass ratio. Trapezoidally-shaped electrodes can be used to increase the maximum ion energy at which ion traces can be prevented from overlapping. Finally, a Multi-Pinhole TPS is proposed as a possible solution to obtain angularly-resolved ion spectra using TPS. The three modifications to the TPS design discussed here can also be implemented simultaneously, providing a compact diagnostic capable of providing angularly-resolved, high-energy resolution spectra of laser-driven multi-species ion sources.

\section{Acknowledgments}

The authors acknowledge funding from EPSRC [EP/J002550/1-Career Acceleration Fellowship held by S. K., EP/L002221/1, EP/K022415/1 and EP/J500094/1. 


\section{References}

[1] A. Macchi, M. Borghesi, and M. Passoni, "Ion acceleration by superintense laser-plasma interaction," Reviews of Modern Physics, vol. 85, no. 2, p. 751, 2013.

[2] S. Wilks, A. Langdon, T. Cowan, M. Roth, M. Singh, S. Hatchett, M. Key, D. Pennington, A. MacKinnon, and R. Snavely, "Energetic proton generation in ultra-intense laser-solid interactions," Physics of Plasmas, vol. 8, p. 542, 2001.

[3] J. Fuchs, P. Antici, E. d'Humières, E. Lefebvre, M. Borghesi, E. Brambrink, C. Cecchetti, M. Kaluza, V. Malka, M. Manclossi, et al., "Laser-driven proton scaling laws and new paths towards energy increase," Nature Physics, vol. 2, no. 1, pp. 48-54, 2005.

[4] L. Robson, P. Simpson, R. Clarke, K. Ledingham, F. Lindau, O. Lundh, T. McCanny, P. Mora, D. Neely, C.-G. Wahlström, et al., "Scaling of proton acceleration driven by petawatt-laser-plasma interactions," Nature Physics, vol. 3, no. 1, pp. 58-62, 2006.

[5] S. Gitomer, R. Jones, F. Begay, A. Ehler, J. Kephart, and R. Kristal, "Fast ions and hot electrons in the laser-plasma interaction,” Physics of Fluids (1958-1988), vol. 29, no. 8, pp. 2679-2688, 1986.

[6] S. Wilks, A. Langdon, T. Cowan, M. Roth, M. Singh, S. Hatchett, M. Key, D. Pennington, A. MacKinnon, and R. Snavely, "Energetic proton generation in ultra-intense laser-solid interactions," Physics of Plasmas (1994-present), vol. 8, no. 2, pp. 542-549, 2001.

[7] F. Nürnberg, M. Schollmeier, E. Brambrink, A. Blažević, D. Carroll, K. Flippo, D. Gautier, M. Geissel, K. Harres, B. Hegelich, et al., "Radiochromic film imaging spectroscopy of laser-accelerated proton beams," Review of scientific instruments, vol. 80, no. 3, p. 033301, 2009.

[8] R. Clarke, P. Simpson, S. Kar, J. Green, C. Bellei, D. Carroll, B. Dromey, S. Kneip, K. Markey, P. McKenna, et al., "Nuclear activation as a high dynamic range diagnostic of laser-plasma interactions," Nuclear Instruments and Methods in Physics Research Section A: Accelerators, Spectrometers, Detectors and Associated Equipment, vol. 585, no. 3, pp. 117-120, 2008.

[9] R. Fleischer, P. Price, and R. Walker, "Ion explosion spike mechanism for formation of charged-particle tracks in solids," Journal of applied Physics, vol. 36, no. 11, pp. 3645-3652, 1965.

[10] S. Kar, M. Borghesi, L. Romagnani, S. Takahashi, A. Zayats, V. Malka, S. Fritzler, and A. Schiavi, "Analysis of latent tracks for mev protons in cr-39," Journal of applied physics, vol. 101, no. 4, p. $044510,2007$.

[11] P. Bolton, M. Borghesi, C. Brenner, D. Carroll, C. De Martinis, F. Fiorini, A. Flacco, V. Floquet, J. Fuchs, P. Gallegos, et al., "Instrumentation for diagnostics and control of laser-accelerated proton (ion) beams," Physica Medica, vol. 30, no. 3, pp. 255-270, 2014.

[12] J. J. Thomson, "Bakerian lecture: Rays of positive electricity," Proceedings of the Royal Society of London. Series A, vol. 89, no. 607, pp. 1-20, 1913.

[13] T. Esirkepov, M. Borghesi, S. Bulanov, G. Mourou, and T. Tajima, "Highly efficient relativistic-ion generation in the laser-piston regime," Physical Review Letters, vol. 92, no. 17, pp. 175003-175003, 2004.

[14] A. Robinson, M. Zepf, S. Kar, R. Evans, and C. Bellei, "Radiation pressure acceleration of thin foils with circularly polarized laser pulses," New Journal of Physics, vol. 10, no. 1, p. 013021, 2008.

[15] S. Kar, K. Kakolee, B. Qiao, A. Macchi, M. Cerchez, D. Doria, M. Geissler, P. McKenna, D. Neely, J. Osterholz, et al., "Ion acceleration in multispecies targets driven by intense laser radiation pressure," Physical Review Letters, vol. 109, no. 18, p. 185006, 2012. 
[16] J. Bin, W. Ma, H. Wang, M. Streeter, C. Kreuzer, D. Kiefer, M. Yeung, S. Cousens, P. Foster, B. Dromey, et al., "Ion acceleration using relativistic pulse shaping in near-critical-density plasmas," Physical review letters, vol. 115, no. 6, p. 064801, 2015.

[17] L. Yin, B. Albright, B. Hegelich, and J. Fernández, "Gev laser ion acceleration from ultrathin targets: The laser break-out afterburner," Laser and Particle Beams, vol. 24, no. 02, pp. 291-298, 2006.

[18] L. Yin, B. Albright, K. Bowers, D. Jung, J. Fernández, and B. Hegelich, “Three-dimensional dynamics of breakout afterburner ion acceleration using high-contrast short-pulse laser and nanoscale targets," Physical Review Letters, vol. 107, no. 4, p. 045003, 2011.

[19] D. Jung, B. Albright, L. Yin, D. Gautier, R. Shah, S. Palaniyappan, S. Letzring, B. Dromey, H.-C. Wu, T. Shimada, R. Johnson, M. Roth, J. Fernandez, D. Habs, and B. Hegelich, "Beam profiles of proton and carbon ions in the relativistic transparency regime," New Journal of Physics, vol. 15, p. 123035, 2013.

[20] A. Alejo, S. Kar, A. Tebartz, H. Ahmed, S. Astbury, D. Carroll, J. Ding, A. Higginson, P. McKenna, D. Neely, N. Neumann, G. Scott, F. Wagner, M.Roth, and M. Borghesi, "Diagnosis of high-energy, multi-species ion sources using a modified Thomson parabola spectrometer," Review of Scientific Instruments, vol. 87, no. 8, p. 083304, 2016.

[21] D. Gwynne, S. Kar, D. Doria, H. Ahmed, M. Cerchez, J. Fernandez, R. Gray, J. Green, F. Hanton, D. MacLellan, P. McKenna, Z. Najmudin, D. Neely, J. Ruiz, A. Schiavi, M. Streeter, M. Swantusch, O. Willi, M. Zepf, and M. Borghesi, "Modified Thomson spectrometer design for high energy, multi-species ion sources," Review of Scientific Instruments, vol. 85, no. 3, p. 033304, 2014.

[22] D. Jung, R. Hörlein, D. Kiefer, S. Letzring, D. Gautier, U. Schramm, C. Hübsch, R. Öhm, B. Albright, J. Fernandez, et al., "Development of a high resolution and high dispersion Thomson parabola," Review of Scientific Instruments, vol. 82, no. 1, p. 013306, 2011.

[23] K. Harres, M. Schollmeier, E. Brambrink, P. Audebert, A. Blažević, K. Flippo, D. Gautier, M. Geißel, B. Hegelich, F. Nürnberg, et al., "Development and calibration of a thomson parabola with microchannel plate for the detection of laser-accelerated mev ions," Review of Scientific Instruments, vol. 79, no. 9, p. 093306, 2008.

[24] F. Schillaci, M. Maggiore, A. Velyhan, G. Cirrone, G. Cuttone, D. Margarone, G. P. Palumbo, P. Pisciotta, D. Rifuggiato, F. Romano, et al., "Calibration and energy resolution study of a high dispersive power thomson parabola spectrometer with monochromatic proton beams," Journal of Instrumentation, vol. 9, no. 10, p. T10003, 2014.

[25] G. A. P. Cirrone, A. Tramontana, G. Candiano, M. Carpinelli, S. Cavallaro, M. Cutroneo, G. Cuttone, C. De Martinis, D. Giove, G. Korn, et al., "A new thomson spectrometer for high energy laser-driven beams diagnostic,” Journal of Instrumentation, vol. 9, no. 08, p. T08001, 2014.

[26] D. Carroll, P. Brummitt, D. Neely, F. Lindau, O. Lundh, C.-G. Wahlström, and P. McKenna, “A modified Thomson parabola spectrometer for high resolution multi-MeV ion measurements-Application to laser-driven ion acceleration," Nuclear Instruments and Methods in Physics Research Section A: Accelerators, Spectrometers, Detectors and Associated Equipment, vol. 620, no. 1, pp. 23-27, 2010.

[27] J. Cobble, K. Flippo, D. Offermann, F. Lopez, J. Oertel, D. Mastrosimone, S. Letzring, and N. Sinenian, "High-resolution thomson parabola for ion analysis," Review of Scientific Instruments, vol. 82, no. 11, p. 113504, 2011.

[28] J. Morrison, C. Willis, R. Freeman, and L. Van Woerkom, "Design of and data reduction from 
compact thomson parabola spectrometers," Review of Scientific Instruments, vol. 82, no. 3, p. 033506, 2011.

[29] F. Consoli, R. De Angelis, A. Bonasera, J. Sura, P. Andreoli, G. Cristofari, M. Cipriani, G. Di Giorgio, F. Ingenito, M. Barbarino, et al., "Study on a compact and adaptable thomson spectrometer for laser-initiated $11 \mathrm{~b}$ (p, $\alpha$ ) 8be reactions and low-medium energy particle detection," Journal of Instrumentation, vol. 11, no. 05, p. C05010, 2016.

[30] "Fujifilm imaging plates." http://www. fujifilm.com.

[31] M. Mori, M. Kando, A. S. Pirozhkov, Y. Hayashi, A. Yogo, N. Yoshimura, K. Ogura, M. Nishiuchi, A. Sagisaka, S. Orimo, M. Kado, A. Fukumi, Z. Li, S. Nakamura, A. Noda, and H. Daido, "New detection device for Thomson parabola spectrometer for diagnosis of the laser-plasma ion beam," Plasma and Fusion Research, vol. 1, pp. 042-042, 2006.

[32] A. Mancic, J. Fuchs, P. Antici, S. Gaillard, and P. Audebert, "Absolute calibration of photostimulable image plate detectors used as (0.5-20," Review of Scientific Instruments, vol. 79, no. 7, pp. 073301-073301, 2008.

[33] I. Choi, C. Kim, J. Sung, I. Kim, T. Yu, S. Lee, Y. Jin, K. Pae, N. Hafz, and J. Lee, "Absolute calibration of a time-of-flight spectrometer and imaging plate for the characterization of laser-accelerated protons," Measurement Science and Technology, vol. 20, no. 11, p. 115112, 2009.

[34] A. Alejo, S. Kar, H. Ahmed, A. Krygier, D. Doria, R. Clarke, J. Fernandez, R. Freeman, J. Fuchs, A. Green, et al., "Characterisation of deuterium spectra from laser driven multi-species sources by employing differentially filtered image plate detectors in thomson spectrometers," Review of Scientific Instruments, vol. 85, no. 9, p. 093303, 2014.

[35] C. Freeman, G. Fiksel, C. Stoeckl, N. Sinenian, M. Canfield, G. Graeper, A. Lombardo, C. Stillman, S. Padalino, C. Mileham, et al., "Calibration of a Thomson parabola ion spectrometer and fujifilm imaging plate detectors for protons, deuterons, and alpha particles," Review of Scientific Instruments, vol. 82, no. 7, pp. 073301-073301, 2011.

[36] T. Bonnet, M. Comet, D. Denis-Petit, F. Gobet, F. Hannachi, M. Tarisien, M. Versteegen, and M. Aleonard, "Response functions of imaging plates to photons, electrons and 4he particles," Review of Scientific Instruments, vol. 84, no. 10, p. 103510, 2013.

[37] D. Doria, S. Kar, H. Ahmed, A. Alejo, J. Fernandez, M. Cerchez, R. J. Gray, F. Hanton, D. A. MacLellan, P. McKenna, Z. Najmudin, D. Neely, L. Romagnani, J. A. Ruiz, G. Sarri, C. Scullion, M. Streeter, M. Swantusch, O. Willi, M. Zepf, and M. Borghesi, "Calibration of bas-tr image plate response to high energy (3-300 mev) carbon ions," Review of Scientific Instruments, vol. 86, no. 12, p. 123302, 2015.

[38] R. Prasad, D. Doria, S. Ter-Avetisyan, P. Foster, K. Quinn, L. Romagnani, C. Brenner, J. Green, P. Gallegos, M. Streeter, D. Carroll, O. Tresca, N. Dover, C. Palmer, J. Schreiber, D. Neely, Z. Najmudin, P. McKenna, M. Zepf, and M. Borghesi, "Calibration of Thomson parabolaâĂŤmcp assembly for multi-mev ion spectroscopy," Nuclear Instruments and Methods in Physics Research Section A: Accelerators, Spectrometers, Detectors and Associated Equipment, vol. 623, no. 2, pp. 712-715, 2010.

[39] T. W. Jeong, P. Singh, C. Scullion, H. Ahmed, K. Kakolee, P. Hadjisolomou, A. Alejo, S. Kar, M. Borghesi, and S. Ter-Avetisyan, "Experimental evaluation of the response of micro-channel plate detector to ions with 10s of mev energies," Review of Scientific Instruments, vol. 87, no. 8, p. 083301, 2016. 
[40] S. Kar, A. Green, H. Ahmed, A. Alejo, A. Robinson, M. Cerchez, R. Clarke, D. Doria, S. Dorkings, J. Fernandez, et al., "Beamed neutron emission driven by laser accelerated light ions," New Journal of Physics, vol. 18, no. 5, p. 053002, 2016.

[41] J. F. Ziegler, M. Ziegler, and J. Biersack, “\{SRIM $\}$ - the stopping and range of ions in matter (2010)," Nuclear Instruments and Methods B, vol. 268, pp. 1818 - 1823, 2010.

[42] C. Danson, P. Brummitt, R. Clarke, J. Collier, B. Fell, A. Frackiewicz, S. Hancock, S. Hawkes, C. Hernandez-Gomez, P. Holligan, M. Hutchinson, A. Kidd, W. Lester, I. Musgrave, D. Neely, D. Neville, P. Norreys, D. Pepler, C. Reason, W. Shaikh, T. Winstone, R. Wyatt, and B. Wyborn, "Vulcan petawatt - an ultra-high-intensity interaction facility," Nuclear Fusion, vol. 44, no. 12, p. S239, 2004.

[43] A. Krygier, J. Morrison, S. Kar, H. Ahmed, A. Alejo, R. Clarke, J. Fuchs, A. Green, D. Jung, A. Kleinschmidt, et al., "Selective deuterium ion acceleration using the vulcan petawatt laser," Physics of Plasmas (1994-present), vol. 22, no. 5, p. 053102, 2015.

[44] W. Mróz, P. Norek, A. Prokopiuk, P. Parys, M. Pfeifer, L. Laska, M. Stöckli, D. Fry, and K. Kasuya, "Method of processing ion energy distributions using a thomson parabola ion spectrograph with a microchannelplate image converter camera," Review of Scientific Instruments, vol. 71, no. 3, pp. 1417-1420, 2000.

[45] E. Breschi, M. Borghesi, D. Campbell, M. Galimberti, D. Giulietti, L. Gizzi, L. Romagnani, A. Schiavi, and O. Willi, "Spectral and angular characterization of laser-produced proton beams from dosimetric measurements," Laser and Particle Beams, vol. 22, no. 04, pp. 393-397, 2004.

[46] N. Dover, C. Palmer, M. Streeter, H. Ahmed, B. Albertazzi, M. Borghesi, D. Carroll, J. Fuchs, R. Heathcote, P. Hilz, et al., "Buffered high charge spectrally-peaked proton beams in the relativistic-transparency regime,” New Journal of Physics, vol. 18, no. 1, p. 013038, 2016.

[47] H. Chen, A. Hazi, R. van Maren, S. Chen, J. Fuchs, M. Gauthier, S. Le Pape, J. Rygg, and R. Shepherd, "An imaging proton spectrometer for short-pulse laser plasma experimentsa)," Review of Scientific instruments, vol. 81, no. 10, p. 10D314, 2010.

[48] D. Jung, R. Hörlein, D. Gautier, S. Letzring, D. Kiefer, K. Allinger, B. Albright, R. Shah, S. Palaniyappan, L. Yin, et al., "A novel high resolution ion wide angle spectrometer," Review of Scientific Instruments, vol. 82, no. 4, p. 043301, 2011.

[49] L. Senje, M. Yeung, B. Aurand, S. Kuschel, C. Rödel, F. Wagner, K. Li, B. Dromey, V. Bagnoud, P. Neumayer, et al., "Diagnostics for studies of novel laser ion acceleration mechanisms," Review of Scientific Instruments, vol. 85, no. 11, p. 113302, 2014.

[50] S. Ter-Avetisyan, M. Schnürer, P. Nickles, W. Sandner, T. Nakamura, and K. Mima, "Correlation of spectral, spatial, and angular characteristics of an ultrashort laser driven proton source," Physics of Plasmas (1994-present), vol. 16, no. 4, p. 043108, 2009.

[51] S. Ter-Avetisyan, L. Romagnani, M. Borghesi, M. Schnürer, and P. Nickles, "Ion diagnostics for laser plasma experiments," Nuclear Instruments and Methods in Physics Research Section A: Accelerators, Spectrometers, Detectors and Associated Equipment, vol. 623, no. 2, pp. 709-711, 2010.

[52] "Simion website." http://simion.com/. 